\title{
Continuation treatment of major depressive disorder: is there a case for duloxetine?
}

This article was published in the following Dove Press journal:

Drug Design, Development and Therapy

II February 2010

Number of times this article has been viewed

\section{Trevor R Norman James S Olver}

Department of Psychiatry, University of Melbourne, Austin Hospital, Heidelberg, Victoria, Australia
Correspondence:Trevor R Norman Department of Psychiatry, University of Melbourne, Austin Hospital, Heidelberg 3084, Victoria, Australia Email trevorrn@unimelb.edu.au

\begin{abstract}
Duloxetine is a serotonin-noradrenaline reuptake inhibitor with established efficacy for the short-term treatment of major depressive disorder. Efficacy in continuation treatment (greater than six months of continuous treatment) has been established from both open and placebocontrolled relapse prevention and comparative studies. Seven published studies were available for review and showed that in both younger and older populations (aged more than 65 years) the acute efficacy of duloxetine was maintained for up to one year. Response to treatment was based on accepted criteria for remission of depression and in continuation studies remission rates were greater than $70 \%$. Comparative studies showed that duloxetine was superior to placebo and comparable to paroxetine and escitalopram in relapse prevention. Importantly a study of duloxetine in patients prone to relapse of major depressive disorder showed that the medication was more effective than placebo in this difficult to treat population. Side effects of duloxetine during continuation treatment were predictable on the basis of the known pharmacology of the drug. In particular there were no significant life-threatening events which emerged with continued use of the medication. On the other hand vigilance is required since the data base on which to judge very rare events is limited by the relatively low exposure to the drug. Duloxetine has established both efficacy and safety for continuation treatment but its place as a first-line treatment of relapse prevention requires further experience. In particular further comparative studies against established agents would be useful in deciding the place of duloxetine in therapy.
\end{abstract}

Keywords: major depression, duloxetine, continuation treatment, placebo studies, relapse prevention, clinical trials

\section{Introduction}

It is estimated by the World Health Organization that major depression affects about 121 million people across the globe. ${ }^{1}$ Some projections suggest that by the year 2020 depression will be among the highest ranked causes of disability, second only to ischemic heart disease. Depression tends to be a recurrent disorder. Indeed it has been estimated that up to $85 \%$ of patients with depression experience a depressive relapse. ${ }^{2}$ Furthermore patients who are left with residual symptoms of depression have an increased risk of recurrence and of future episodes of depression. ${ }^{3}$ Recent treatment outcome and long-term follow-up studies of patients with an index episode of illness show that complete elimination of symptoms ie, remission should be the primary goal of any form of treatment. Elimination of symptoms has been shown to be associated with diminished risk of relapse. ${ }^{4}$

The goal of continuation treatment is to eliminate residual symptoms, restore social functioning and to prevent relapse of depression. ${ }^{5}$ The degree to which 
antidepressant medications are capable of achieving each of these goals is mixed and is perhaps most readily demonstrated for relapse prevention. This can be demonstrated by alternative methodological approaches, such as continuing open treatment to determine rates of relapse. Clearly a more rigorous evaluation method is to randomly assign patients who have achieved remission of depression, in a double-blind manner, to either placebo or to continue the dose of medication that initially achieved the desired response. Time to an a priori-defined relapse criterion can then be measured in both groups and the statistical differences assessed from Kaplan-Meier survival analysis. Using this general methodological approach the effectiveness for relapse prevention of most classes of antidepressants has been demonstrated.

In a meta-analysis of 31 studies involving 4410 subjects continuing treatment with antidepressants reduced the chance of relapse by $70 \%$ compared with cessation of treatment. ${ }^{6}$ The treatment effect persisted for up to 36 months. Thus tricyclic antidepressants have been clearly demonstrated to prevent relapse. Controlled clinical trials have established the effectiveness of amitriptyline, ${ }^{7,8}$ imipramine $^{9-11}$ nortriptyline ${ }^{12}$ and desipramine ${ }^{13}$ as well as the monoamine oxidase inhibitor phenelzine. ${ }^{14,15}$ The duration of these studies varied from 16 weeks to three years and each demonstrated a superiority of the active drug over placebo in preventing relapse. Similar double-blind evaluations have been performed for the selective serotonin reuptake inhibitors (SSRIs) fluoxetine ${ }^{16,17}$ sertraline, ${ }^{18,19}$ paroxetine, ${ }^{20-22}$ citalopram, ${ }^{23,24}$ and escitalopram. ${ }^{25}$ Generally SSRIs are treatments of choice for continuation therapy due to their better tolerability compared to tricyclic antidepressants and monoamine oxidase (MAO) inhibitors. ${ }^{26}$ With the development of the serotonin-noradrenaline reuptake inhibitor (SNRI) antidepressants, there is an emerging literature on their use for continuation therapy. Thus milnacipran has demonstrated efficacy in the continuation phase of treatment superior to placebo. ${ }^{27}$ Recently, venlafaxine has demonstrated superiority over placebo for relapse prevention at both one year ${ }^{28}$ and two year ${ }^{29,30}$ follow-up periods.

The need for continuation therapy of major depression to maintain clinical response has been clearly demonstrated. Relapse of depression has been shown to occur in patients remitted from depression when switched to placebo or if treatment is discontinued. It is incumbent then on any new treatment to establish its credentials in this area. Acute treatment trials are often the focus of attention for the purposes of registration of new medications with the appropriate authorities. Efficacy of new treatments in continuation therapy is often poorly established.

\section{Overview of pharmacology and pharmacokinetics of duloxetine}

Duloxetine is a dual serotonin (5HT) and noradrenaline (NE) reuptake inhibitor ${ }^{31,32}$ with little affinity for a range of other neurotransmitter receptors. ${ }^{33}$ Human in vitro studies in healthy volunteers suggest that both serotonin and noradrenaline reuptake may account for the antidepressant activity of duloxetine. ${ }^{34,35}$ Duloxetine exhibits linear pharmacokinetics with an elimination half-life in the range of 7 to 27 hours. ${ }^{36}$ It is highly protein-bound ( $>90 \%)$ and extensively distributed to tissues. Duloxetine is eliminated through hepatic metabolism involving CYP2D6 and CYP1A2. ${ }^{37}$

\section{Duloxetine in acute treatment of major depression}

The efficacy of duloxetine in acute treatment of major depressive disorder has been reviewed recently. ${ }^{38}$ Duloxetine is more effective than placebo and at least as effective as other established antidepressants (fluoxetine, paroxetine, escitalopram, venlafaxine). A database of 17 randomized controlled acute trials of duloxetine in adult MDD was subjected to meta-analysis with random effects modeling. ${ }^{38}$ Response rates (reduction of baseline HAM-D by $\geq 50 \%$ ) for duloxetine were $48.8 \%$ to $59.6 \%$ and for placebo were $35.0 \%$ to $42.2 \%$. This gave an average of $40 \%$ superiority of duloxetine over placebo. In the published trials remission rates for duloxetine ranged from $23 \%$ to $54 \%$, based on the last-observation-carried-forward analysis, compared to $15 \%$ to $30 \%$ for placebo-treated patients. Analysis of comparative data did not establish any meaningful clinical differences in efficacy from SSRI antidepressants.

\section{Efficacy of duloxetine in continuation treatment}

Clinical trials undertaken to examine the efficacy of duloxetine in continuation treatment of major depressive disorder are summarized in Table 1. Six main studies were conducted in patients who met either Diagnostic and Statistical Manual of Mental Disorders, 4th edition (DSM-IV) or DSM-IV-TR criteria for the disorder. (A seventh study, also summarized in Table 1, was an analysis of a subgroup of elderly patients from a previous trial). Duloxetine was used for up to 52 weeks in one study while the remaining studies were conducted over 24 or 26 weeks of treatment. 
Table I Continuation studies of duloxetine in major depressive disorder

\begin{tabular}{|c|c|c|c|c|c|}
\hline Design & $\begin{array}{l}\text { Subjects, age, } \\
\text { dose, length } \\
\text { of treatment }\end{array}$ & $\begin{array}{l}\text { Outcome } \\
\text { measures }\end{array}$ & $\begin{array}{l}\text { Statistical } \\
\text { analysis }\end{array}$ & Efficacy & Authors \\
\hline Open label & $\begin{array}{l}928 F, 35 I M \\
(18-87 \text { y) } 120 \mathrm{mg} / \text { day } \\
52 \text { weeks }\end{array}$ & $\begin{array}{l}\text { HDRS BDRS } \\
\text { CGI-S } \\
\text { PGI-I Sheehan }\end{array}$ & MMRM-ANOVA & $\begin{array}{l}\text { Change from baseline } \\
(P<0.00 \mathrm{I}) \text { all measures; } \\
81.8 \% \text { remission at end-point }\end{array}$ & Raskin et al ${ }^{39}$ \\
\hline Open label** & $\begin{array}{l}72 F, 29 M \\
(65-87 y)\end{array}$ & $\begin{array}{l}\text { HDRS BDRS } \\
\text { CGI-S PGI-I } \\
\text { Sheehan }\end{array}$ & MMRM-ANOVA & $\begin{array}{l}\text { Change from baseline on all } \\
\text { measures }(P<0.00 \mathrm{I}) ; 72.3 \% \\
\text { remission at end-point }\end{array}$ & Wohlreich et al ${ }^{40}$ \\
\hline $\begin{array}{l}\text { Double blind run-in; } \\
\text { continuation in responders }\end{array}$ & $\begin{array}{l}267 F, 100 M(\geq 18 \text { y) } \\
\text { DUL } 80 \mathrm{mg} / \text { day } \\
\text { DUL } 120 \mathrm{mg} / \text { day PAR } \\
20 \mathrm{mg} / \text { day PBO } 26 \text { weeks }\end{array}$ & $\begin{array}{l}\text { HDRS MADRS } \\
\text { HARS CGI-S } \\
\text { PGI-IVAS }\end{array}$ & Kaplan-Meier & $\begin{array}{l}\text { Longer time to loss of } \\
\text { response for all active drugs } \\
\text { compared to PBO }(P<0.05)\end{array}$ & Detke et $\mathrm{a}^{45}$ \\
\hline $\begin{array}{l}\text { Open label } 12 \text { week run-in; } \\
\text { responders randomized } \\
\text { to DUL or PBO }\end{array}$ & $\begin{array}{l}202 \mathrm{~F}, 76 \mathrm{M}(\geq 18 \mathrm{y}) \\
60 \mathrm{mg} / \text { day } 26 \text { weeks }\end{array}$ & HDRS CGI-SVAS & $\begin{array}{l}\text { Kaplan-Meier; } \\
\text { ANCOVA }\end{array}$ & $\begin{array}{l}\text { Relapse } 23 \% \text { DUL vs } 39 \% \text { PBO } \\
(P<0.05) \text {; time to relapse } \\
\text { shorter on PBO }(P<0.005)\end{array}$ & Perahia et $\mathrm{al}^{43}$ \\
\hline $\begin{array}{l}\text { Double-blind, parallel } \\
\text { group study }\end{array}$ & $\begin{array}{l}212 F, 83 M(18-73 \text { y) } \\
\text { DUL } 60 \mathrm{mg} / \text { dayESC } \\
20 \mathrm{mg} / \text { day } 24 \text { weeks }\end{array}$ & $\begin{array}{l}\text { MADRS HDRS } \\
\text { CGI-S CGI-I HARS } \\
\text { Sheehan }\end{array}$ & ANCOVA & $\begin{array}{l}\text { Mean change in MADRS } 21.7 \\
\text { DUL, } 23.4 \text { ESC (NS); } \\
\text { Significant decline in MADRS } \\
\text { over time }(P<0.05)\end{array}$ & Wade et $\mathrm{a}^{46}$ \\
\hline $\begin{array}{l}\text { Open label extension } \\
\text { study }\end{array}$ & $\begin{array}{l}\text { I26F, } 5 \text { IM }(43.7 \pm I I .6 \mathrm{y}) \\
\text { DUL } 60-120 \mathrm{mg} / \text { day } \\
\text { Up to } 623 \text { days }\end{array}$ & HDRS CGI-S & No formal analysis & $\begin{array}{l}\text { HDRS did rise above } 7 \\
\text { throughout the extension } \\
\text { phase. }\end{array}$ & Dunner et $\mathrm{al}^{4 \mathrm{l}}$ \\
\hline $\begin{array}{l}\text { Double-blind, placebo } \\
\text { controlled study }\end{array}$ & $\begin{array}{l}29 I F, I 22 \mathrm{M}(47.4 \pm 13.0 \mathrm{y}) \\
\text { DUL } 60-120 \mathrm{mg} / \text { day } \\
\text { PBO } 52 \text { weeks }\end{array}$ & $\begin{array}{l}\text { HDRS CGI-S PGI-I } \\
\text { SF-36 Sheehan }\end{array}$ & $\begin{array}{l}\text { Kaplan-Meier; } \\
\text { MMRM ANOVA }\end{array}$ & $\begin{array}{l}\text { Improvements in depression } \\
\text { ratings from open phase } \\
\text { maintained through } \\
\text { continuation for DUL; DUL } \\
\text { patients had longer time to } \\
\text { relapse than PBO }(P<0.00 \mathrm{I}) \text {. }\end{array}$ & Perahia et a $\left.\right|^{44}$ \\
\hline
\end{tabular}

Notes: *Patients in the studies met either DSM-IV or DSM-IV-TR criteria for major depressive disorder; $* *$ This study was a reanalysis of data from the Raskin and colleagues ${ }^{39}$ study for the elderly (aged $>65$ years) population.

Abbreviations: DUL, duloxetine; ESC, escitalopram; PAR, paroxetine; PBO, placebo; BDRS, Beck depression rating scale; CGI-I, Clinical Global Impression Scale-Improvement; CGI-S, Clinical Global Impression Scale-Severity; HDRS, Hamilton Depression Rating Scale; HARS, Hamilton Anxiety Rating Scale; PGI-I, Patient Global Impression ScaleImprovement; Sheehan, Sheehan disability scales;VAS, visual analog scale.

In one naturalistic study duloxetine was administered for up to 623 days.

\section{Open evaluations}

The efficacy of duloxetine administered over a maximum period of 52 weeks for the treatment of major depressive disorder (MDD) was investigated in an open-label trial. ${ }^{39} \mathrm{To}$ be eligible for admission to the study patients had to meet the DSM-IV criteria for MDD and have a Clinical Global Impressions Scale (CGI-S) score of $>3$ at screening and baseline visits. There were no eligibility criteria in terms of the severity of depression at baseline as rated with the Hamilton Depression Rating Scale (HAM-D). The study was conducted at eight sites in North and South America. Patients received duloxetine 40 to $60 \mathrm{mg}$ twice daily with the dose adjusted according to the physician's judgment of tolerability. Efficacy of treatment was assessed using the CGI-S, HAM-D 17 item scale, the Beck Depression Inventory, and the Patient Global Impression-Improvement scale (PGI-I). Quality of life was evaluated using the Sheehan Disability Scale. Outcomes were assessed at weeks 6,28 , and 52 or earlier if the patient discontinued treatment. PGI-I and CGI-S were evaluated at all visits. Response and remission were defined on the basis of a decline from baseline of the HAM-D score of $50 \%$ or more (response) or $\leq 7$ (remission). A total of 1279 patients entered the study (72.6\% female) of whom $553(43.2 \%)$ completed 52 weeks of treatment. After two weeks of treatment those patients unable to tolerate $80 \mathrm{mg} /$ day of duloxetine were discontinued. Of the patients who did not complete the 52 weeks of treatment, $17 \%$ dropped out due to adverse events, $6.6 \%$ due to noncompliance, and $5.9 \%$ for lack of efficacy. The remainder of the patients who dropped out of the study did so for personal reasons $(10.2 \%)$, protocol violations (3.1\%), or were lost to follow-up $(9.3 \%)$, or for other reasons 
(5.7\%). The main adverse events associated with dropping out of the study were nausea, somnolence, vomiting, diarrhea, hypomania, and hypertension. The HAM-D scale showed a highly statistically significant decline from baseline at all measurement times $(P<0.001$; repeated measures, analysis of variance [ANOVA]). The change in rating was also clinically significant falling from a mean of 22.5 at baseline to 9.3 at week 6, 5.9 at week 28, and 5.0 at week 52. Factors of the HAM-D scales were also significantly reduced over time as were the Beck Depression Inventory scale and the Sheehan disability sub-scales. Rates of response and remission, estimated using the last-observation-carried-forward (LOCF) were $71 \%$ and $60 \%$, respectively. Probability estimates were performed for response and remission with $62.9 \%$ and $50.8 \%$ observed at week 6 , respectively. These increased with time in treatment to $84.3 \%$ and $75.6 \%$ at week 28 and $89.1 \%$ and $81.8 \%$ at week 52 . Although an open evaluation, the study suggests that continuation treatment of depression with duloxetine is capable of achieving remission for a substantial number of patients.

For patients aged 65 years or more who took part in this open evaluation a subgroup analysis was undertaken. ${ }^{40}$ At the beginning of the continuation treatment phase there were 101 patients (mean age 71.9 years). In general the clinical efficacy results were not different from those obtained when the total group was analyzed. Thus there were statistically significant improvements in the HAM-D 17-item total score from baseline as well as in both the clinician (CGI-S) and patient (PGI-I) rated measures of improvement. Response rates (defined as noted above for this study) at weeks 6, 28, and 52 were $62.9 \%, 84.9 \%$, and $89.4 \%$, respectively. The corresponding remission rates (see earlier definition for the study) at these times were $41.4 \%, 69.8 \%$, and $72.3 \%$, respectively. In this older sub-group, duloxetine maintained clinical response in the long term and was not distinguishable from the responses observed in patients aged 18 to 64 years. In this elderly sub-group discontinuation due to adverse events in this sub-group were $26.7 \%$ of patients which is somewhat higher than the group as a whole (17\%). The main adverse events associated with withdrawal were somnolence $(4.0 \%)$, dizziness $(3.0 \%)$, diarrhea $(2.0 \%)$, hypertension $(2.0 \%)$, and vomiting $(2.0 \%)$. Another $5.0 \%$ of patients withdrew due to noncompliance while $9.9 \%$ discontinued due to personal or other reasons. Most discontinuations (66.7\%) occurred within the first two weeks of initiation of treatment.

Further evidence for the efficacy of duloxetine in continuation treatment was obtained from a naturalistic study. ${ }^{41}$ In this multicenter trial, patients met DSM-IV criteria for
MDD and had a HAM-D total score of $\geq 15$ and a CGI-S score of $\geq 4$ at baseline. Subjects received 30 or $60 \mathrm{mg} /$ day of duloxetine in the first week of treatment and thereafter the dose could be titrated to a maximum of $120 \mathrm{mg} /$ day. Subjects who completed 12 weeks of treatment were eligible to enter the continuation phase. The results of the open 12-week study were reported separately. ${ }^{42}$ In this phase of the study response was defined as a reduction of $50 \%$ or more from baseline of the HAM-D score and remission as a HAM-D total score $<7$. These criteria were used throughout the continuation phase. Patients were followed on a monthly basis after the acute phase of the study. A total of 177 patients commenced the extension phase and the mean duration of treatment in this phase was 305 days with a range of 4 to 623 days. Throughout the extension phase the mean HAM-D score remained below 7 . Thus the data provide evidence for the continuing efficacy of duloxetine over extended periods. For the patients entering the extension phase, 119 began as remitters of depression, 27 as responders, and 31 as nonresponders. Generally remitters remained in remission throughout the extension phase with only 16 patients (13\%) becoming nonresponders. For initial nonresponders, 16 patients $(52 \%)$ became remitters or responders while for the initial responders, 11 patients $(41 \%)$ became remitters, and 9 (33\%) became nonresponders. During the continuation phase of treatment 21 patients $(11.9 \%)$ discontinued treatment due to adverse events. Of these patients 11 had events which either worsened during the continuation phase or were ongoing and did not improve. For the other patients, new events leading to discontinuation were creatine phosphokinase (CPK) increased, hypertension, hypomania, intentional self injury, rash, suicidal ideation, weight gain, nephrolithiasis, and babesiosis.

\section{Placebo-controlled, relapse prevention studies}

Prevention of relapse of major depressive disorder provides superior evidence for the efficacy of a treatment in this condition. Thus a relapse prevention study was performed in outpatients who had responded to $60 \mathrm{mg}$ daily of duloxetine in an open evaluation. ${ }^{43}$ In the open phase of the study 533 patients were treated with duloxetine and those who responded at week 12 were randomly allocated in a double-blind manner to either continue duloxetine or to receive placebo. For the open phase response to medication was defined as a HAM-D score of $\leq 12$ and a CGI-S score of $\leq 2$. Furthermore, patients should also no longer have met DSM-IV criteria for MDD. Patients were then treated for up to 26 weeks. Re-emergence of depressive symptoms was defined as a HAM-D score 
$\geq 12$, while relapse was defined as a CGI-S score of $\geq 2$ points compared to that at week 12 and meeting the MINI criteria for MDD at two consecutive visits at least two weeks apart. Of the original 533 patients, 136 continued to receive duloxetine, and 142 to receive placebo. The primary efficacy analysis compared the time from randomization to relapse between the two groups using the log-rank test. The two groups for the continuation phase of treatment were well matched in terms of age, gender distribution and severity of illness on both the HAM-D and CGI-S scales. Patients receiving duloxetine took a statistically significantly longer time to relapse than those receiving placebo $(P<0.005$, Kaplan-Meier). Based on the protocol criteria for relapse $28.5 \%$ of placebo-treated patients relapsed compared to $17.4 \%$ of duloxetine-treated patients over this time frame $(P<0.05)$. When assessed by the investigator alone to have relapsed, $43.1 \%$ of placebo compared to $21.9 \%$ of duloxetine patients met clinical judgment criteria $(P<0.001)$. Changes in the HAM-D scale over the continuation period were evaluated as a secondary criterion of efficacy. Patients treated with duloxetine were less likely than those treated with placebo to have a deterioration in their total 17-item score $(P<0.01 ;$ ANOVA). In addition to the total HAM-D score factors derived from the scale also showed statistical and clinically relevant superiority of duloxetine over placebo. The study shows that duloxetine maintains its clinical efficacy in the treatment of MDD over an extended time frame. In the continuation phase there were no statistically significant differences between duloxetine- and placebotreated patients for the rates of adverse events. A total of five patients discontinued duloxetine in the extension phase due to adverse events: anxiety, cholelithiasis, spastic colon, ejaculation failure, and gastroesophageal reflux were reported by one patient each. A similar number of placebo-treated patients discontinued treatment. In the group randomized to duloxetine, one patient discontinued due to lack of efficacy, 11 due to their own decision, and a further 16 patients due to protocol violations or were lost to follow-up.

A more recent study reported on prevention of depressive recurrences in a group of subjects meeting DSM-IV criteria for MDD. ${ }^{44}$ An additional criterion for entry into the study was that patients should have had at least three episodes of depression in the past five years. In this multicentre trial after open treatment for up to 34 weeks with 60-120 mg/day of duloxetine (administered once daily), patients meeting response criteria were randomly assigned to placebo or duloxetine for up to 52 weeks in a double-blind maintenance phase. Response criteria were HAM-D score $\leq 9$, CGI-S $\leq 2$, and no longer meeting DSM-IV criteria for MDD. The primary outcome measure was the time to recurrence of a depressive episode. Criteria for recurrence were based on any of the following being met: CGI-S score $\geq 4$; meeting DSM-IV criteria for MDD; three consecutive visits meeting re-emergence criteria (ie, CGI-S $\geq 4$ but not fulfilling DSM criteria for MDD) or 10 total re-emergence visits; withdrawal due to lack of efficacy. Of the 514 patients who initially entered the study, 288 were eligible for the maintenance phase. Both time to depressive recurrence and the time to a $50 \%$ increase in depressive symptoms (based on HAM-D total score) was significantly longer in duloxetinetreated than in placebo-treated patients $(P<0.001)$. In the double-blind phase $33.1 \%$ of patients receiving placebo experienced recurrence of depression whereas $14.1 \%$ of patients receiving duloxetine had a recurrence of depression $(P<0.001)$. It can be concluded that duloxetine is useful in the prevention of recurrence of depressive episodes in patients prone to recurrence. The proportion of patients who discontinued in the maintenance phase due to adverse events was $2.1 \%$ in the placebo group and $4.1 \%$ in the duloxetine group. In this phase of the study there were no statistically significant differences between groups for treatment emergent adverse events.

\section{Placebo-controlled, comparative relapse prevention studies}

Duloxetine was compared to paroxetine and placebo in the acute and long-term treatment of major depressive disorder. ${ }^{45}$ Following an 8-week double-blind, placebo-controlled study patients who had responded to treatment were continued for up to six months on blinded medication. Response was defined as a $\geq 30 \%$ decline in baseline HAM-D total score. All patients met DSM-IV criteria for MDD which was confirmed by the MINI interview schedule. In the initial study 367 patients were randomly allocated on a 1:1:1:1 basis to receive duloxetine $80 \mathrm{mg} /$ day, duloxetine $120 \mathrm{mg} /$ day, paroxetine $20 \mathrm{mg}$ /day, or placebo (approximately 90 per group). The primary efficacy variable was the HAM-D 17-item scale with secondary outcome measures obtained from the factors of the HAM-D scale, the Montgomery-Åsberg Depression Rating Scale (MADRS) scale, HAM-A, Visual Analog Scale (VAS), CGI-S, and PGI-I scales. In addition the Sheehan Disability Scale and the Somatic Symptom Inventory were also assessed at baseline, weeks 1, 2, 4, 6, and 8 . In the acute phase all active treatments were superior to placebo for all outcome measures. For the primary outcome variable, difference between baseline and end-point HAM-D total score, 
both doses of duloxetine and paroxetine were superior to placebo ( $P<0.001$; analysis of covariance [ANCOVA]). Subsequent to the acute phase a total of 273 patients entered the continuation phase. During this phase of treatment loss of response was defined as the first visit at which the HAM-D showed a less than $30 \%$ reduction from the acute baseline score. This criterion varies from that used in the majority of studies where a score on the HAM-D scale is usually taken as a cut-off for relapse. Given that the mean HAM-D at baseline across the groups was $\sim 20$ then the cut-off for relapse would have been a HAM-D score of $\sim 14$. This is a more lenient criterion than in other trials but on the other hand allows for individual differences in starting baseline scores. Statistically significant differences between treatments were assessed by means of a Kaplan-Meier estimate. All three active treatment groups had a longer time to loss of response than did placebo ( $P<0.005$; log-rank test). Remission (HAM-D total score $\leq 7$ ) rates at the end of the acute study were $46 \%$ for duloxetine $80 \mathrm{mg} /$ day, $52 \%$ for duloxetine $120 \mathrm{mg} /$ day, $44 \%$ for paroxetine, and $30 \%$ for placebo. It is not clear for the study whether these remission (or response) rates increased with continued treatment. Nevertheless the study does support continuing efficacy of duloxetine during ongoing treatment. The proportion of patients who discontinued due to adverse events was similar across all four treatment groups: $6.9 \%$ placebo; $4.3 \%$ duloxetine $80 \mathrm{mg} /$ day; $6.7 \%$ duloxetine $120 \mathrm{mg} /$ day; $2.9 \%$ paroxetine.

A similar study compared the efficacy of acute and continuation treatment with duloxetine and escitalopram in outpatients with MDD. ${ }^{46}$ Patients in this study met DSM-IVTR criteria for MDD as confirmed by the MINI interview. In addition patients met the severity criteria of a MADRS total score of $\geq 26$ and a CGI-S score of $\geq 4$. Patients were randomly allocated, on a double-blind basis and a 1:1 ratio, to treatment with either duloxetine $60 \mathrm{mg} /$ day or escitalopram $20 \mathrm{mg} /$ day. The duration of the study was 24 weeks. Efficacy was assessed at baseline and weeks 1, 2, 4, 8, 12, 16,20 , and 24 using the MADRS scale as the primary outcome measure. The mean change in MADRS score from baseline to week 24 based on the intent to treat population was used to assess efficacy. The last observation carried forward was used to account for missing data. An analysis of covariance was used to assess statistical significance of the change. Secondary efficacy measures included the HAM-D scale, CGI-S, and the HAM-A scale. Remission was defined as a MADRS $\leq 12$ or HAM-D $\leq 7$. Response was defined as a decline from baseline of $>50 \%$ in the MADRS or HAM-D score. A total of 294 patients were enrolled in the study (151 duloxetine, 143 escitalopram) whereas the intention-to-treat (ITT) data set was 287 patients (146 duloxetine, 141 escitalopram). There were few statistically significant differences between the two drugs at any time point throughout the study. At week 8 remission rates were $48 \%$ for duloxetine and $56 \%$ for escitalopram (nonsignificant [NS]) while at week 24 the respective rates were $70 \%$ and $73 \%$ (NS). The study again supports the notion of the efficacy of duloxetine in the long-term treatment of MDD. Significantly more patients withdrew from the duloxetine $(17 \%)$ than the escitalopram $(9 \%)$ as a result of adverse events. Nausea, agitation, and insomnia were the main events leading to withdrawal from the study.

\section{Safety of duloxetine}

Apart from efficacy the major concern with continuation treatment of any medication is its safety and the occurrence of serious adverse events. The safety of duloxetine has been the subject of reviews using pooled data. ${ }^{47,48}$ In each of the studies described above the general tolerability of duloxetine was evaluated. The emergence of adverse events serious enough to warrant the concern of authorities and which may lead to the withdrawal of an agent are not usually apparent until the post-marketing phase. There is no indication from the clinical studies so far conducted with duloxetine that any rare life-threatening events have occurred.

\section{Adverse events}

For patients who underwent treatment beyond the short-term, new emergent adverse events were generally diminished compared to the acute phase of treatment. For each of the trials reviewed emergent adverse events during the continuation phase are summarized in Table 2. The consistency with which events were reported varied from study to study but in most cases new adverse effects which emerged in the continuation phase were tabulated. The exception was the study by Perahia et $\mathrm{al}^{43}$ where no tabulation of events was available but a summary of events leading to discontinuation of treatment was provided.

In the one year open-label trial, ${ }^{39}$ the incidence of all events occurring within the first eight weeks of treatment were markedly reduced over the next 44 weeks. The most common side effect in the first eight weeks of treatment was nausea, experienced by $31.8 \%$ of patients. From week 9 to 52 the incidence of nausea was reduced to $3.4 \%$ of patients treated. Reductions of a similar magnitude were noted for other adverse events, eg, somnolence $27.7 \%$ to $2.8 \%$, insomnia $24.9 \%$ to $7.3 \%$, headache $22.4 \%$ to $10.0 \%$, dry mouth $22.1 \%$ to $2.7 \%$, 
Table 2 Emergent events during continuation treatment with duloxetine

\begin{tabular}{|c|c|c|c|c|c|c|}
\hline Event & Raskin & Wohlreich & Dunner & Detke $^{\neq}$ & Wade* & Perahia (2) \\
\hline Nausea & 3.4 & 0 & NR & NR & 31.4 & NR \\
\hline Somnolence & 2.8 & 1.0 & NR & NR & 1.3 & NR \\
\hline Insomnia & 7.3 & 7.9 & 5.6 & 2.7 & 12.6 & 4.8 \\
\hline Headache & 10.0 & 5.9 & I.I & 2.7 & 16.6 & 8.9 \\
\hline Dry mouth & 2.7 & 4.0 & NR & NR & 13.2 & NR \\
\hline Constipation & 3.3 & 5.0 & NR & NR & 8.6 & NR \\
\hline Dizziness & 6.4 & 5.0 & 10.7 & 2.7 & 15.9 & 3.4 \\
\hline Sweatiness & 4.3 & 4.0 & 1.7 & NR & 7.3 & NR \\
\hline Diarrhea & 3.8 & 5.9 & NR & 4.0 & 7.3 & NR \\
\hline Tremor & 1.3 & 2.0 & 1.7 & NR & NR & NR \\
\hline Anxiety & 7.3 & 3.0 & 2.2 & 2.7 & NR & NR \\
\hline Fatigue & 3.4 & 4.0 & 2.2 & NR & 11.3 & 5.5 \\
\hline Decreased appetite & 0.9 & 1.0 & NR & NR & NR & NR \\
\hline Anorexia & 1.2 & 3.0 & 1.1 & NR & NR & NR \\
\hline Vomiting & 2.6 & 3.0 & 3.9 & NR & 7.3 & NR \\
\hline Back pain & NR & 2.0 & 7.3 & NR & NR & 8.9 \\
\hline Other pain & NR & 2.0 & 4.5 & 2.7 & NR & NR \\
\hline
\end{tabular}

Notes: For the study by Perahia and colleagues, ${ }^{43}$ a list of adverse events and incidences was not reported. ${ }^{\neq}$Data from treatment with 120 mg/day of duloxetine; ${ }^{*}$ Adverse events in the extension phase were not separated from the acute phase.

Abbreviation: NR, no reports for this individual event in the published data.

constipation $18.8 \%$ to $3.3 \%$. Tolerance to the side effects of duloxetine clearly develops with continued treatment. Serious adverse events were reported by 64 patients in this study and included suicidal ideation (7), suicide attempt (7), accident (3), hip fracture (3), angina (2), anxiety (2), cholelithiasis (2), confusion (2), depression aggravated (2), mania (2), and overdose (2). Seventeen percent of patients discontinued treatment due to an adverse event during the continuation phase. From this same study data for patients aged over 65 years was reanalyzed with respect to the adverse event profile. ${ }^{40}$ In this older sub-population $(n=101)$ all treatment emergent adverse events were diminished in weeks 9 to 52 compared to weeks 1 to 8 . Thus nausea was reduced from $28.7 \%$ to $0 \%$, dizziness from $26.7 \%$ to $5.0 \%$, somnolence $21.8 \%$ to $1.0 \%$, dry mouth from $15.8 \%$ to $4.0 \%$, and insomnia from $14.9 \%$ to $7.9 \%$. Older patients tended to report statistically significantly less insomnia and headache than younger patients. The incidence of other adverse events was not different between elderly and young patients. There were single reports of syncope and postural hypotension in the elderly subgroup, while two patients experienced falls during the study.

In the open evaluation conducted by Dunner and colleagues, data were analyzed for new or worsening adverse events during the extension phase. ${ }^{41}$ Few patients reported new clinically significant adverse events. The main new events reported were fatigue $(9.0 \%)$ and hyperhidrosis (4.5\%). The time to onset of these events was not analyzed.

In the six month continuation study, ${ }^{45}$ no new treatment emergent adverse events were reported by more than $5 \%$ of patients in the continuation phase. The main treatment emergent events during continuation were diarrhea, headache, nausea, insomnia, anxiety, dizziness, flatulence, and pain. These were reported by less than $4 \%$ of all patients treated and were dose dependent. Three patients died in this phase of the study due to suicide (1 duloxetine $120 \mathrm{mg} /$ day; 1 placebo) or pulmonary edema (duloxetine $80 \mathrm{mg} /$ day).

Statistically significant differences in the rate of adverse events reported between duloxetine and placebo were not observed during a prevention of relapse study conducted over 26 weeks of treatment. ${ }^{43}$ No further details of the adverse events were reported in this study except for events leading to discontinuation of study drug as noted above.

In a double-blind, dose escalation study patients with DSM-IV major depressive disorder were treated with duloxetine according to a dosage regimen that was increased to $120 \mathrm{mg} /$ day over the first three weeks and then stabilized for a further four weeks. ${ }^{49}$ This period was followed by an open-label, six-week stabilization phase during which the duloxetine dose was adjusted to the optimal maintenance dose. Following the stabilization period patients could 
continue treatment depending on clinical need. The primary objective of the study was to assess safety and tolerability of duloxetine. A total of 81 patients entered the extension phase. During this phase the main emergent adverse events were upper respiratory tract infection (13.1\%), headache (10.7\%), insomnia (10.7\%), anxiety (9.5\%), increased weight (9.5\%), nasopharyngitis $(8.3 \%)$, constipation $(7.1 \%)$, hyperhidrosis (7.1\%), abnormal dreams (6.0\%), and sinusitis $(6.0 \%)$. The rate of discontinuation due to adverse events in this phase of the study was $11.9 \%$ whereas $21.4 \%$ discontinued due to lack of efficacy, $15.5 \%$ were lost to follow-up, and $15.5 \%$ due to patient decision or protocol violations.

Treatment emergent adverse events occurred with a similar frequency in placebo- and duloxetine-treated patients during relapse prevention over 52 weeks. ${ }^{44}$ Headache $(8.9 \%)$, back pain (8.9\%), nasopharyngitis (6.2\%), influenza (3.4\%), insomnia (4.8\%), dizziness (3.4\%), and fatigue (5.5\%) were reported as events associated with duloxetine administration. The difference in incidence for the same events in placebotreated patients was not statistically significant.

Adverse events emerging specifically in the continuation phase of treatment with either duloxetine or escitalopram were not available, as the data were compared across the entire 24 week period. ${ }^{46}$ The only statistically significant difference between treatments was for insomnia which occurred more frequently with duloxetine than with escitalopram $(12.6 \%$ vs $4.9 \%)$. It is not clear whether this difference was due to the early phase or continuation phase of treatment.

In summary, duloxetine appears to be well tolerated during extended periods of use with tolerance developing to the side effects of medication over the course of treatment. The main emergent events likely to occur with continued use appear to be hyperhidrosis, fatigue, somnolence, insomnia, and dizziness.

\section{Clinical laboratory data}

Short-term treatment with duloxetine has been associated with small increases in liver enzyme values. ${ }^{50}$ Alkaline phosphatase, alanine aminotransferase (ALT) and aspartate aminotransferase (AST) were all increased compared to placebo-treated patients. In the continuation studies reviewed here there was sporadic reporting of data for clinical laboratory tests. Statistically significant changes from baseline to last observation for some laboratory parameters were reported in the open 12 month study. ${ }^{39}$ However it was noted that the mean changes were small compared to baseline values and was regarded as not clinically significant. The laboratory values outside of the normal range were relatively few.
Furthermore, there did not appear to be any temporal pattern of the emergence of these events. Abnormal values tended to decline with treatment and mean changes from baseline to week 52 were close to zero. None of the changes in values were associated with clinical symptoms. Similar findings and conclusions were drawn for the elderly subgroup of patients in this study analyzed separately. ${ }^{40}$ Small mean changes in laboratory parameters which were not considered clinically meaningful were also reported in a placebo-controlled sixmonth continuation study. ${ }^{45}$

No clinically significant or persistent changes in clinical laboratory results were observed in patients treated with duloxetine for prevention of relapse of major depression. ${ }^{43} \mathrm{In}$ the continuation phase of this study elevations of ALT $(n=7)$ and AST $(n=4)$ were noted for duloxetine-treated patients. The elevated levels resolved spontaneously. No other specific laboratory parameter abnormalities were noted in the report. Similarly three patients had elevated hepatic parameters (three times the upper limit of normal) in a dose escalation study of duloxetine. ${ }^{49}$ No other abnormalities from the extension phase of this study were reported. Statistically significant differences in the percentage of subjects exhibiting laboratory abnormalities between placebo and duloxetine at any time during a relapse prevention trial were observed only for bilirubin. ${ }^{44}$ Abnormally high levels were observed in the placebo group (3.9\% vs $0 \%$ in the duloxetine group). The mean change from baseline to last observation for ALT was significantly different between placebo and duloxetine $(-0.38 \pm 12.5$ vs $2.52 \pm 15.4 \mathrm{U} / \mathrm{L}(P<0.05)$ but not for any other analyte at any time point. Information for laboratory tests was not available from the other studies reviewed here.

\section{Vital signs and cardiovascular effects}

The cardiovascular profile was a particular focus of the majority of continuation trials with duloxetine. Mean changes in supine and standing blood pressure (BP) from baseline to last observation did not change statistically or clinically significantly during 52 weeks treatment with duloxetine. ${ }^{39}$ There were small increases in supine and standing pulse $(1.5$ and $1.8 \mathrm{bpm})$ in this study. An analysis of patients who were hypertensive at baseline compared to those who were nonhypertensive was also performed. Mean changes in supine and standing diastolic and systolic BP did not differ between hypertensive and nonhypertensive subjects. During treatment, 46 of 1039 patients who were normotensive at baseline met criteria for hypertension. Of these patients 23 returned to baseline BP during continued treatment with the drug. Of the 1039 normotensive patients only two had a 
sustained hypertensive episode (systolic BP $\geq 180 \mathrm{mmHg}$ or diastolic $\geq 110 \mathrm{mmHg}$ ). There were no significant changes in electrocardiogram (ECG) intervals from baseline. Mean change from baseline to last observation in the corrected QT interval (QTc) was $0.52 \mathrm{msec}$, not different from zero. One female and one male patient had a treatment emergent QTc interval $>450 \mathrm{msec}$. Similar findings were reported for the analysis of the elderly sub-group of patients in this study. ${ }^{40}$ The rate of potentially clinically significant low values for standing BP was 5.2\% and for all other BP and pulse readings was $<2.5 \%$. Changes in cardiac intervals detected by ECG were not clinically significant.

No statistically significant mean changes in baseline to endpoint heart rate, systolic and diastolic BP were noted in the continued treatment with duloxetine 80 and $120 \mathrm{mg} /$ day for up to six months. ${ }^{45}$ Mean changes in the QTc interval were not significantly altered from baseline to endpoint. No patients had QTc intervals $\geq 500 \mathrm{msec}$. The cardiovascular profile of duloxetine was reportedly similar to that of placebo. ${ }^{43} \mathrm{Small}$, but statistically significant differences, were noted for the change in diastolic BP between duloxetine- $(60 \mathrm{mg} /$ day $)$ and placebo-treated patients $(+2 \mathrm{mmHg}$ vs $-1 \mathrm{mmHg} ; P<0.005)$ during this 26 week continuation study. There were no statistically significant mean changes in systolic BP and heart rate over the same period. Increases of $1.4 \mathrm{mmHg}$ and $1.1 \mathrm{mmHg}$ were noted for supine systolic and diastolic BP respectively in a continuation trial of duloxetine for up to two years. ${ }^{49}$ These changes were not statistically significant when compared to baseline. Heart rate was increased by $3.1 \mathrm{bpm}(P<0.001$ compared to baseline). Mean increase in systolic BP, adjusted for baseline, after 1.5 years treatment with duloxetine was between 1.3 and $2.2 \mathrm{mmHg} .{ }^{41}$ For diastolic BP the adjusted mean was between -0.1 and $0.5 \mathrm{mmHg}$. Two patients met criteria for sustained hypertension. For pulse the adjusted mean increases were between 1.7 and $4.2 \mathrm{bpm}$.

A more extensive analysis of BP, pulse, and ECG changes was performed in a relapse prevention study with duloxetine compared to placebo. ${ }^{44}$ Mean changes in BP and pulse did not differ significantly between placebo- and duloxetine-treated patients. The mean ( \pm standard deviation $[\mathrm{SD}]$ ) change in systolic BP was $1.5( \pm 1.0) \mathrm{mmHg}$ and diastolic BP was -0.2 $( \pm 0.7) \mathrm{mmHg}$ for duloxetine-treated patients. Criteria for sustained elevations of BP were noted in three duloxetineand two placebo-treated subjects. The QTc interval was not different between groups and there were no potentially clinically significant QTc increases at any time in the study. The mean change in QTc for duloxetine patients was $1.3( \pm 1.6)$ milliseconds. Heart rate was increased by $0.2( \pm 0.95) \mathrm{bpm}$.
Patients treated with duloxetine for up to 24 weeks had nonstatistically significant increases of $1.2 \mathrm{mmHg}$ in systolic and $0.3 \mathrm{mmHg}$ in diastolic $\mathrm{BP}{ }^{45}$ Heart rate was increased by $2.7 \mathrm{bpm}$ from baseline.

Confirming the generally safe cardiovascular profile of duloxetine have been some studies specifically designed to address the issue as well as meta-analyses of the clinical trial database for major depression and other conditions. A particular issue with many psychotropic medications has been the association with sudden cardiac death, probably as a result of prolongation of the corrected QT interval (QTc). ${ }^{51}$ In healthy female volunteers supra-therapeutic exposures to duloxetine were compared in the presence and absence of $400 \mathrm{mg}$ moxifloxacin, a drug known to prolong QTc interval. ${ }^{52}$ Mean QTc interval was not prolonged and in fact decreased from baseline. At the doses used in this study (160 mg and $200 \mathrm{mg} \mathrm{bd}$ ) duloxetine is unlikely to affect cardiac conduction in healthy subjects. Data from short term clinical trials suggest that the mean change in the QRS width of the electrocardiogram was not clinically significant. ${ }^{47}$

A meta-analysis of the cardiovascular safety of duloxetine was performed in more than 8000 patients treated with the drug or placebo for up to 36 weeks. ${ }^{47}$ This safety profile was based on vital signs, ECGs and emergent events potentially related to cardiovascular effects of the drug. Increases in heart rate and decreases in QTc interval were more likely to occur in duloxetine-treated patients. However such changes as were observed were not regarded as clinically significant. A prolongation of the QTc interval to $>500$ msecs occurred in one duloxetine-treated patient Mean increases in systolic and diastolic BP were $0.65 \mathrm{mmHg}$ and $0.88 \mathrm{mmHg}$ respectively for duloxetine-treated patients. There was no evidence for a sustained increase in BP associated with the use of duloxetine. Adverse events potentially related to the cardiovascular system such as palpitations, tachycardia, orthostatic hypotension, hypertension and peripheral edema occurred in duloxetine- and placebotreated patients to similar extents. These events occurred with a frequency less than $2 \%$.

Duloxetine at the doses used in the clinical trials would appear to have a favorable cardiovascular safety profile.

\section{Body weight}

After 52 weeks of treatment with duloxetine, a statistically significant weight increase of $2.4 \mathrm{~kg}$ was noted from baseline. ${ }^{39}$ Using the LOCF data set the mean change in weight was $1.1 \mathrm{~kg}$. Analysis of the elderly sub-group 
within this study showed a mean weight loss of $0.1 \mathrm{~kg}$ using baseline to last observation. ${ }^{40}$ On the other hand, using the mixed model repeated measures analysis there was a mean weight gain of $0.3 \mathrm{~kg}$. Potentially clinically significant weight loss was experienced by three patients while potentially clinically significant weight gain was experienced by six patients. Weight increased significantly in all treatment groups during continuation treatment of up to six months. ${ }^{45}$ For placebo the mean change in weight was $0.4( \pm 15.2) \mathrm{kg}$, for duloxetine $80 \mathrm{mg} /$ day was $1.4( \pm 15.1) \mathrm{kg}$, duloxetine $120 \mathrm{mg} /$ day $1.9( \pm 19.2) \mathrm{kg}$ and for paroxetine $20 \mathrm{mg} /$ day was $1.6( \pm 16.1) \mathrm{kg}$. Mean change in body was $0.9 \mathrm{~kg}$ in patients treated with duloxetine for up to two years. ${ }^{49}$ Similarly during 1.5 years of treatment mean weight gain depended on whether patients were drug-naïve or had received previous treatments. ${ }^{41}$ Thus for drug-naïve patients mean weight gain was $2.54 \mathrm{~kg}$ compared with $0.4 \mathrm{~kg}$ for previously treated patients. A mean weight gain of 0.88 $( \pm 0.36) \mathrm{kg}$ in duloxetine-treated patients was not statistically different from that of placebo-treated patients during relapse prevention. ${ }^{44}$ Post hoc analysis of the proportion of patients with weight gain $\geq 7 \%$ of baseline showed no differences between duloxetine and placebo.

The outcome for weight gain or loss was not reported in the other continuation studies.

It can be expected that long-term treatment will result in weight gain for some patients. This may be due in part to effects of medication but may also be due to the resolution of depressive symptoms when appetite is regained.

\section{Sexual function}

Sexual dysfunction, which may emerge or worsen during the course of treatment, represents a significant clinical problem and is common to most, if not all, antidepressant medications. ${ }^{53}$ Treatment emergent sexual dysfunction in the relapse prevention study was assessed using the Arizona Sexual Experience Scale (ASEX). ${ }^{44}$ Mean scores on the scale tended to improve from baseline for both men and women, but not statistically significantly. There were no differences between duloxetine and placebo for changes from baseline. In the other studies sexual dysfunction was recorded as a reported side effect of medication. Decreased libido (4.1\%), ejaculation failure (2.7\%), and erectile dysfunction $(2.5 \%)$ were reported as side effects of duloxetine during a 52 week continuation study. ${ }^{39}$ For loss of libido the relative incidence between male and female subjects was not distinguished. Erectile dysfunction (2.4\%), delayed ejaculation $(1.2 \%)$, decreased libido (1.2\%), and loss of libido $(1.2 \%)$ were reported in a two-year continuation study. 49

The incidence of treatment emergent sexual dysfunction with duloxetine was assessed by pooling data from four double-blind, placebo-controlled clinical trials. ${ }^{54}$ Data from two continuation treatment studies, up to 26 weeks, was available for the analysis. Sexual function was assessed using the ASEX administered at baseline and endpoint. In contrast to the experience in the short term, there was no difference between duloxetine and placebo for the incidence of sexual dysfunction (39.4\% vs $35.3 \%$ ) in the continuation phase. In an eight-month double-blind, placebo-controlled study in patients with MDD sexual functioning was assessed using the Changes in Sexual Functioning Questionnaire (CSFQ). ${ }^{55}$ In the short-term (up to eight weeks) both duloxetine and escitalopram had significantly higher incidences of sexual dysfunction than placebo, for patients who did not meet criteria at baseline for sexual dysfunction. At eight months of treatment, emergent sexual side effects were $33.3 \%$ for duloxetine, $43.6 \%$ escitalopram, and $25.0 \%$ for placebo. These differences were not statistically significantly different. Patients who recovered from MDD consistently showed improvements in sexual functioning whereas those who did not improve tended to show a worsening of function, as measured by the CSFQ. In this study there were no statistically significant differences between duloxetine and escitalopram discontinuation rates for sexual dysfunction.

While it should be expected that sexual dysfunction is likely to be a significant adverse event for many patients, this will be confounded by the outcome of the depressive episode itself.

\section{Discontinuation emergent events}

Abrupt discontinuation was studied after long-term treatment when patients were assessed two weeks after ceasing medication. ${ }^{39}$ The main effects which were reported were dizziness $(8.3 \%)$, anxiety $(4.3 \%)$, nausea $(4.2 \%)$, headache $(3.1 \%)$, insomnia $(2.9 \%)$, and irritability $(2.6 \%)$. In the elderly sub-group analysis for this study the adverse events recorded in this phase were dizziness $(8.9 \%)$, anxiety $(7.9 \%)$, headache $(5.0 \%)$, and insomnia $(5.0 \%) .{ }^{40}$ Discontinuation adverse events were reported for $23.0 \%$ of duloxetine-treated patients following abrupt discontinuation of drug after a 52-week double-blind, maintenance treatment. ${ }^{44}$ Three serious events, convulsions, hypertension, and hypertensive crisis, were experienced by one patient who discontinued duloxetine. 
Discontinuation was not specifically reported in the other studies reviewed as they were designed to assess the efficacy of ongoing treatment.

Both the type and the rate of discontinuation emergent adverse events appear to be similar in the continuation studies as those observed after short-term treatment. It seems likely that duloxetine should not be abruptly discontinued but should be tapered on withdrawal.

\section{Other events}

The emergence of suicidal ideation, suicide, and the switch into hypomania or mania are significant problems with the use of antidepressant drugs in both the short- and long-term. Some cases of suicide attempts during therapy with duloxetine have been reported. ${ }^{56}$ Significantly in long term trials there have been reports of suicide attempts and the emergence of suicidal ideation..$^{39}$ While these have been estimated to be not significantly different from placebo, at least from metaanalysis of short term studies ${ }^{57}$ suicide and the development of suicidal ideation is an ever present risk in the treatment of depression.

Data from a pooled analysis of eight acute phase tri$\mathrm{als}^{58}$ showed that the emergence of mania or hypomania in patients treated with duloxetine was low $(0.2 \%)$. Whether this translates to continuation treatment is not clear, but two cases of mania were reported during one year of treatment. ${ }^{39}$ The emergence of manic episodes in other continuation trials was not specifically reported.

\section{Conclusions}

Duloxetine has demonstrated efficacy in the treatment of major depressive disorder in continuation studies for up to one year or more. The medication maintains its shortterm effectiveness during continued treatment. Importantly controlled studies suggest that duloxetine is able to prevent relapse significantly better than placebo, which attests to the efficacy of the drug for continuation treatment. Furthermore, a study in patients prone to relapse of their depressive episodes showed duloxetine to be effective in maintenance treatment. Although the current data base for continuation treatment is relatively circumscribed, there is sufficient evidence to be assured that in patients who initially respond to the medication, efficacy, measured in terms of remission of an index episode of depression, is continued in the longer term. Tolerance to the majority of side effects tends to develop with continued treatment. However, as with all medications continued pharmacovigilance needs to be undertaken to monitor for rare, but potentially dangerous, events. On current levels of exposure there is no evidence for these rare life threatening events, but clearly confidence in this statement increases with greater exposure.

\section{Disclosures}

Trevor R Norman has served on advisory boards and/or received support for travel, talks or research from AstraZeneca, Boehringer Ingelheim, Bristol-Myers Squibb, Eli-Lilly, Janssen, Lundbeck, Organon, Pfizer, Servier, and Wyeth in the past five years. James S Olver has received educational and research grants from Astra-Zeneca, Eli-Lilly, and Janssen in the past five years.

\section{References}

1. World Health Organization. Factsheet: Depression 2008. Available from: http://www.who.int/mental_health/management/depression/definition/ en/. Accessed September 11, 2008.

2. Mueller TI, Leon AC, Keller MB, et al. Recurrence after recovery from major depressive disorder during 15 years of observational follow-up. Am J Psychiatry. 1999;156:1000-1006.

3. Judd LL, Akiskal HS, Maser JD, et al. Major depressive disorder: a prospective study of residual subthreshold depressive symptoms as predictor of rapid relapse. J Affect Disord. 1998;50:97-108.

4. Paykel ES, Ramana R, Cooper Z, et al. Prevention of relapse in residual depression an important outcome in depression. Psychol Med. 1995;25:1171-1180.

5. Mann JJ. The medical management of depression. $N$ Engl J Med. 2005;353:1819-1834.

6. Geddes JR, Carney SM, Davies C, et al. Relapse prevention with antidepressant drug treatment in depressive disorders: a systematic review. Lancet. 2003;361:651-653.

7. Coppen A, Ghose K, Montgomery S, Rama RV, Bailey J, Jorgensen A. Continuation therapy with amitriptyline in depression. Br J Psychiatry. 1978;133:28-33.

8. Glen AI, Johnson AL, Shepherd M. Continuation therapy with lithium and amitriptyline in unipolar depressive illness: a randomised doubleblind, controlled trial. Psychol Med. 1984;14:37-50.

9. Kane JM, Quitkin FM, Rifkin A, Ramos L, Nayak DD, Howard A. Lithium carbonate and imipramine in the prophylaxis of unipolar and bipolar II illness: a prospective, placebo-controlled comparison. Arch Gen Psychiatry. 1982;39:1065-1069.

10. Prien RF, Klett CJ, Caffey EM. Lithium carbonate and imipramine in the prevention of affective episodes. Arch Gen Psychiatry. 1973;29: 420-425.

11. Prien RF, Kupfer DJ, Mansky PA. Drug therapy in the prevention of recurrences in unipolar and bipolar affective disorders. Report of the NIMH Collaborative Study Group comparing lithium carbonate, imipramine and a lithium carbonate-imipramine combination. Arch Gen Psychiatry. 1984;41:1096-1104.

12. Alexopoulos GS, Meyers BS, Young RC. Executive dysfunction and long-term outcomes of geriatric depression. Arch Gen Psychiatry. 2000;57:285-290.

13. Kocsis JH, Friedman RA, Markowitz JC. Maintenance therapy for chronic depression. A controlled clinical trial of desipramine. Arch Gen Psychiatry. 1996;53:769-774.

14. Robinson RS, Lerfald SC, Bennett B. Continuation and maintenance treatment of major depression with the monoamine oxidase inhibitor phenelzine: a double-blind placebo-controlled discontinuation study. Psychopharmacol Bull. 1991;27:31-39. 
15. Georgotas A, McCue RE, Cooper TB. A placebo-controlled comparison of nortriptyline and phenelzine in maintenance therapy of elderly depressed patients. Arch Gen Psychiatry. 1989;46:783-786.

16. Montgomery SA, Dufour H, Brion S. The prophylactic efficacy of fluoxetine in unipolar depression. Br J Psychiatry. 1988;3(Suppl): 69-76.

17. Gilaberte I, Montejo AL, de la Gandara J. Fluoxetine in the prevention of depressive recurrences: a double-blind study. J Clin Psychopharmacol. 2001;21:417-424.

18. Doogan DP, Caillard V. Sertraline in the prevention of depression. Br J Psychiatry. 1992;160:217-222.

19. Wilson KC, Mottram PG, Ashworth L, Abou-Saleh MT. Older community residents with depression: long-term treatment with sertraline, double-blind, placebo-controlled study. Br J Psychiatry. 2003;182: 492-497.

20. Eric L. A prospective, double-blind comparative multi-centre study of paroxetine in preventing recurrent major depressive episodes. Biol Psychiatry. 1991;29:11-14.

21. Montgomery SA, Dunbar G. Paroxetine is better than placebo in relapse prevention and the prophylaxis of recurrent depression. Int Clin Psychopharmacol. 1993;8:189-195.

22. Reynolds CF, Dew MA, Pollock BG. Maintenance treatment of major depression in old age. $N$ Engl J Med. 2006;354:1130-1138

23. Hochstrasser B, Isaksen PM, Koponen H. Prophylactic effect of citalopram in unipolar recurrent depression: placebo-controlled study of maintenance therapy. Br J Psychiatry. 2001;178:304-310.

24. Klysner R, Bent-Hansen J, Hansen HL. Efficacy of citalopram in the prevention of recurrent depression in elderly patients: placebo-controlled study of maintenance therapy. Br J Psychiatry. 2002;181:29-35.

25. Kornstein SG, Bose A, Li DY, Saikali KG, Gandhi C. Escitalopram maintenance treatment for prevention of recurrent depression: A randomised, placebo-controlled trial. J Clin Psychiatry. 2006;67: $1767-1775$

26. Hirschfeld RMA, Schatzberg AF. Long-term management of depression. Am J Med. 1994;97(Suppl 6A):33S-38S.

27. Rouillon F, Berdeaux G, Bisserbe JC. Prevention of recurrent depressive episodes with milnacipran: consequences on quality of life. $J$ Affect Disord. 2000;58:171-180

28. Kocsis JH, Thase ME, Trivedi MH, et al. Prevention of recurrent episodes of depression with venlafaxine ER in a 1-year maintenance phase from the PREVENT study. J Clin Psychiatry. 2007;68: 1014-1023.

29. Keller MB, Trivedi MH, Thase ME, et al. The prevention of recurrent episodes of depression with venlafaxine for two years (PREVENT) study: Outcomes from the 2-year and combined maintenance phases. J Clin Psychiatry. 2007;68:1246-1256.

30. Kornstein SG, Kocsis JH, Ahmed S, et al. Assessing the efficacy of 2 years maintenance treatment with venlafaxine extended release $75-225 \mathrm{mg} /$ day in patients with recurrent major depression: a secondary analysis from the PREVENT study. Int Clin Psychopharmacol. 2008;23:357-363.

31. Wong DT. Duloxetine (LY248686): an inhibitor of serotonin and noradrenaline uptake and an antidepressant drug candidate. Exp Opin Invest Drugs. 1998;7:1-9.

32. Kasamo K, Blier P, de Montigny C. Blockade of serotonin and norepinephrine uptake processes by duloxetine: in vitro and in vivo studies in the rat brain. J Pharmacol Exp Ther. 1996;277:278-286.

33. Bymaster FP, Dreshfield-Ahmad LJ, Threkeld PG, et al. Comparative affinity of duloxetine and venlafaxine for serotonin and norepinephrine transporters in vitro and in vivo, human serotonin receptor subtypes and other neuronal receptors. Neuropsychopharmacology. 2001;25: 871-880.

34. Turcotte JE, Debonnel G, de Montigny C, et al. Assessment of the serotonin and norepinephrine reuptake blocking properties of duloxetine in healthy subjects. Neuropsychopharmacology. 2001;24:511-521.
35. Vincent S, Bieck PR, Garland EM, et al. Clinical assessment of norepinephrine transporter blockade through biochemical and pharmacological profiles. Circulation. 2004;109:3202-3207.

36. Sharma A, Goldberg MJ, Cerimele BJ. Pharmacokinetics and safety of duloxetine, a dual-serotonin and norepinephrine reuptake inhibitor. J Clin Pharmacol. 2000;40:161-167.

37. Lantz RJ, Gillespie TA, Rash TJ, et al. Metabolism, excretion and pharmacokinetics of duloxetine in healthy human subjects. Drug Metab Dispos. 2003;31:1142-1150.

38. Girardi P, Pompili M, Innamorati M, et al. Duloxetine in acute major depression: review of comparisons to placebo and standard antidepressants using dissimilar methods. Hum Psychopharmacol. 2009;24: 177-190.

39. Raskin J, Goldstein DJ, Mallinckrodt CH, et al. Duloxetine in the long-term treatment of major depressive disorder. J Clin Psychiatry. 2003;64:1237-1244.

40. Wohlreich MM, Mallinckrodt CH, Watkin JG, et al. Duloxetine for the long-term treatment of major depressive disorder in patients aged 65 years and older: an open-label study. BMC Geriatrics. 2004; 4:11.

41. Dunner DL, Wilson M, Fava M, et al. Long-term tolerability and effectiveness of duloxetine in the treatment of major depressive disorder. Depress Anxiety. 2008;25:E1-E8.

42. Wohlreich MM, Martinez JM, Mallinckrodt CH, et al. An open-label study of duloxetine for the treatment of major depressive disorder. $J$ Clin Psychopharmacol. 2005;25:552-560.

43. Perahia DG, Gilaberte I, Wang F, et al. Duloxetine in the prevention of relapse of major depressive disorder. Double-blind placebo-controlled study. Br J Psychiatry. 2006;188:346-353.

44. Perahia DGS, Maina G, Thase M, et al. Duloxetine in the prevention of depressive recurrences: a randomised, double-blind, placebo controlled study. J Clin Psychiatry. 2009;70:706-716.

45. Detke MJ, Wiltse CG, Mallinckrodt CH, et al. Duloxetine in the acute and long-term treatment of major depressive disorder: a placebo- and paroxetine-controlled trial. Eur Neuropsychopharmacol. 2004;14: 457-470.

46. Wade A, Gembert K, Florea I. A comparative study of the efficacy of acute and continuation treatment with escitalopram versus duloxetine in patients with major depressive disorder. Curr Med Res Opin. 2007;23:1605-1614.

47. Hudson JI, Wohlreich MM, Kajdasz DK, et al. Safety and tolerability of duloxetine in the treatment of major depressive disorder: analysis of pooled data from eight placebo-controlled clinical trials. Hum Psychopharmacol. 2005;20:327-341.

48. Gartlehner G, Thaler K, Hansen RA, Gaynes BN. The general and comparative efficacy and safety of duloxetine: a systematic review and meta-analysis. Drug Saf. 2009;32:1159-1173.

49. Wohlreich MM, Mallinckrodt CH, Prakash BA, et al. Duloxetine for the treatment of major depressive disorder: Safety and tolerability associated with dose escalation. Depress Anxiety. 2007;24:41-52.

50. Frampton JE, Plosker GL. Duloxetine: a review of its use in the treatment of major depressive disorder. CNS Drugs. 2007;21: 581-609.

51. Fermini B, Fossa AA. The impact of drug-induced QT interval prolongation on drug discovery. Nat Rev Drug Discov. 2003;2:439-447.

52. Zhang L, Chappell J, Gonzales CR, et al. QT effects of duloxetine at supratherapeutic doses: A placebo and positive controlled study. J Cardiovasc Pharmacol. 2007;49:146-153.

53. Serretti A, Chiesa A. Treatment emergent sexual dysfunction related to antidepressants: A meta-analysis. J Clin Psychopharmacol. 2009:29:259-266.

54. Delgado PL, Brannan SK, Mallinckrodt CH, et al. Sexual functioning assessed in 4 double-blind, placebo- and paroxetine-controlled trials of duloxetine for major depressive disorder. J Clin Psychiatry. 2005;66:686-692. 
55. Clayton A, Kornstein S, Prakash A, et al. Changes in sexual functioning associated with duloxetine, escitalopram and placebo in the treatment of patients with major depressive disorder. J Sex Med. 2007;4: 917-929.

56. Salem BA, Karem EG. Duloxetine and suicide attempts: a possible relation. Clin Pract Epidemiol Ment Health. 2008;4:18-19.
57. Acharya N, Rosen AS, Polzer JP, et al. Duloxetine: meta-analyses of suicidal behaviours and ideation in clinical trials for major depressive disorder. J Clin Psychopharmacol. 2006;26:587-594.

58. Dunner DL, D'Souza DN, Kajdasz DK, et al. Is treatment-associated hypomania rare with duloxetine: secondary analysis of controlled trials in non-bipolar depression. J Affect Disord. 2005;87:115-119.

\section{Publish your work in this journal}

Drug Design, Development and Therapy is an international, peerreviewed open-access journal that spans the spectrum of drug design and development through to clinical applications. Clinical outcomes, patient safety, and programs for the development and effective, safe, and sustained use of medicines are a feature of the journal, which has also been accepted for indexing on PubMed Central. The manuscript management system is completely online and includes a very quick and fair peer-review system, which is all easy to use. Visit http://www.dovepress.com/testimonials.php to read real quotes from published authors.

Submit your manuscript here: http://www.dovepress.com/drug-design-development-and-therapy-journal 